\title{
Antropología
}

\section{La comunicación interprofesional desde la cultura organizacional de la enfermería asistencial}

\section{Interprofessional communication from the organizational culture of nursing care}

\section{Comunicação interprofissional desde a cultura organizacional de cuidados de enfermagem}

Concepción Lucas García*. Carmen Isabel Gómez García ${ }^{\star *}$. Josefa Mª Antón Hurtado***

* Enfermera asistencial Hospital Rafael Méndez. Profesora asociada Departamento de enfermería de la Universidad de Murcia. Facultad de Ciencias Sociosanitarias de Lorca

** Profesora titular de la Facultad de Enfermería de la Universidad de Murcia.Departamento de enfermería. Facultad de Enfermería de la Universidad de Murcia.

*** Profesora titular de la Facultad de Filosofía de la Universidad de Murcia.Área de Antropología Social. Facultad de Filosofía de la Universidad de Murcia

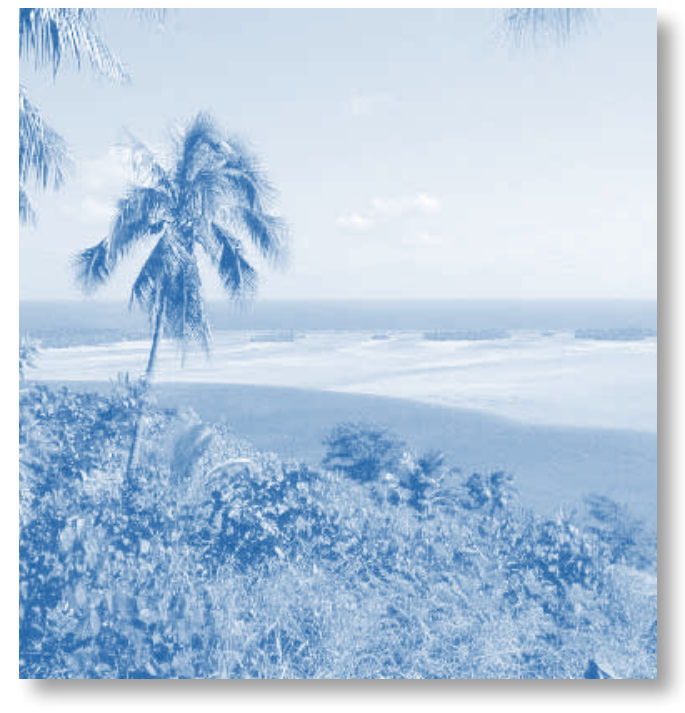

ABSTRACT

Introduction: Good communication between health professionals is recognized as essential for proper patient care, but many authors claim that it remains one of the unfinished business of healthcare organizations.

Objectives: To explore the expectations and ideas nurses have about interprofessional communication in an intensive care unit in a district hospital and establish its relationship to cultural, organizational and personal.

Material and methods: This is a descriptive qualitative methodology, using data collection in-depth semi-structured interviews.

Results: After content analysis identified two categories: expressions of the reality of the communicative interactions between professionals and professional expectations and their proposals for improvement.

Conclusions and Discussion: Each health profession has a different culture that makes each one has its own values and disciplinary point of view of the needs of the patient but also different styles of communication.

It is important to recognize that effective communication is complex and multifactorial, but the leaders should take the concern and interest expressed by professionals to promote iniciatives for the exchange and collaboration.

Key-words: Professional cultures, communication, nursing. Teamwork. 


\section{RESUMO}

Introdução: A comunicação adequada entre os profissionais de saúde é reconhecida como indispensável para um bom atendimento dos pacientes, mas muitos autores garantir que continua a ser um dos negócios inacabados de organizações de saúde.

Objectivos: Explore as expectativas e as idéias que enfermeiras comunicação interdisciplinar em uma unidade de cuidados intensivos hospital regional e estabelecer sua relação com aspectos culturais, organizacionais e pessoais.

Materiais e métodos: Esta é uma pesquisa descritiva com metodologia qualitativa, usando a coleção de dados semi-estruturados entrevistas em profundidade.

Resultados: Após análise de conteúdo foram estabelecidas duas categorias: expressões sobre a realidade das interacções comunicativas entre os profissionais e as expectativas de profissionais e suas sugestões de melhoria.

Conclusões e discussão: cada profissão da saúde tem uma cultura diferente, que faz cada têm seus próprios valores e o ponto de vista disciplinar as necessidades do paciente mas também estilos de comunicação diferentes. É importante reconhecer que uma comunicação eficaz é complexa e multifatorial, mas os líderes devem aproveitar a preocupação e o interesse manifestado por profissionais para promover iniciativas para o intercâmbio e a colaboração.

Palavras-chave: culturas profissionais, comunicação, enfermagem. Trabalho em equipe.

\section{RESUMEN}

Introducción: La comunicación adecuada entre los profesionales de la salud es reconocida como indispensable para un adecuado cui-

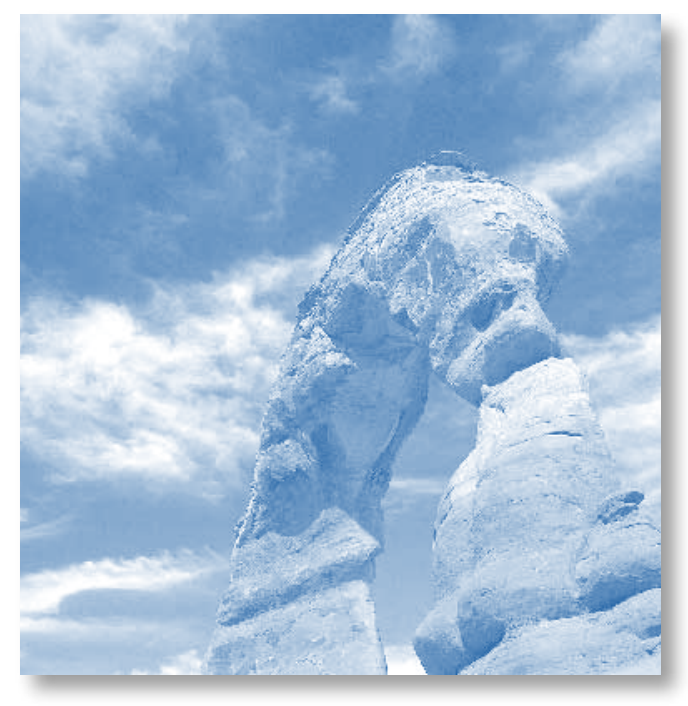

dado de los pacientes, pero numerosos autores aseguran que sigue siendo una de las asignaturas pendientes de las organizaciones sanitarias.

Objetivos: Explorar las expectativas e ideas que tienen los profesionales de enfermería con respecto a la comunicación interprofesional dentro de una unidad de cuidados intensivos de un hospital comarcal y establecer su relación con aspectos culturales, organizacionales y personales.

Material y método: Se trata de una investigación descriptiva con metodología cualitativa, usando para la recolección de datos entrevistas semiestructuradas en profundidad.

Resultados: Tras el análisis de contenido se establecieron dos categorías: expresiones sobre la realidad de las interacciones comunicativas entre los profesionales y expectativas de los profesionales y sus propuestas de mejora.

Conclusiones y Discusión: Cada profesión sanitaria tiene una cultura diferente, que hace que cada uno tenga sus propios valores $y$ punto de vista disciplinario de las necesidades del paciente pero también diferentes estilos de comunicación.

Es importante reconocer que la comunicación efectiva es compleja y multifactorial, pero 
los lideres deberían aprovechar la preocupación y el interés expresados por los profesionales para promover iniciativas para el intercambio y la colaboración.

Palabras clave: Culturas profesionales, comunicación, enfermería. Trabajo en equipo.

\section{INTRODUCCIÓN}

La comunicación adecuada entre los profesionales de la salud es reconocida como indispensable para un adecuado cuidado de los pacientes (Leonard, Graham y Bonacum, 2004.), y relacionando la deficiente comunicación con los errores médicos y la seguridad del paciente (Salas et al, 2009), la calidad de la atención, la eficacia y el rendimiento (Baker, Day y Salas, 2006), así como con la satisfacción y el estrés de los profesionales, y la creación de entornos de trabajo saludables (Clark, 2009). Pero numerosos autores aseguran que sigue siendo una de las asignaturas pendientes de las organizaciones sanitarias (Arizeta, Portillo y Ayestarán, 2001). En el contexto hospitalario y de la atención aguda, constatan que los fallos en la comunicación provocar un aumento de daño al paciente, de la duración de la estancia hospitalaria y del uso de recursos, así como mayor insatisfacción en los profesionales (Zwarenstein y Reeves 2002). Concretamente acentúan su transcendencia en entornos de alta complejidad y dinamismo, como son las Unidad de Cuidados Intensivos (Reader, Flin, Mearns y Cuthbertson, 2009).

La comunicación permite a las personas construir relaciones interpersonales funcionales que les posibilita para trabajar conjuntamente y lograr las metas previstas (Gil y Barrasa, 2003). Presupone dos conceptos el de relación entre un emisor y un receptor, y el de transmisión de información, para ello es ne- cesario establecer unas condiciones idóneas y tener una predisposición para la emisión e intercambio de significados (Cleries y Kronfly, 2000).

Con nuestra investigación hemos querido indagar en los aspectos que pueden estar interviniendo en las interacciones comunicativas entre los profesionales de la salud en una UCI polivalente de 9 camas de un hospital comarcal público de 283 camas, perteneciente a la red de Hospitales del Servicio Murciano de Salud.

\section{OBJETIVOS}

Este trabajo ha tenido como objetivos explorar las expectativas e ideas que tienen los profesionales de enfermería con respecto a la comunicación interprofesional dentro de una unidad de cuidados intensivos de un hospital comarcal y establecer su relación con aspectos culturales, organizacionales y personales, allí presentes.

Pretendemos a través de las opiniones expresadas por los profesionales de enfermería detectar los problemas de comunicación en esta unidad así como las propuestas de mejora que ellos sugieren.

\section{Metodología}

Se ha diseñado un estudio de investigación descriptivo con metodología cualitativa y enfoque etnográfico, usando como técnica para la recolección de datos entrevistas semiestructuradas en profundidad que se realizaron entre abril y junio de 2010 .

Los sujetos de nuestro estudio, son los profesionales de enfermería que trabajan de forma habitual en esta unidad de cuidados intensivos. En la consideración de no realizar más entrevistas se ha tenido en cuenta el principio de saturación teórica (Glaser y Strauss, 1980). 
Se incluyeron en el estudio a enfermeros con una experiencia laboral en la unidad de estudio superior a dos años y con predisposición para colaborar, así como la máxima variabilidad en cuanto a edad, sexo y trayectoria profesional. Se les envió una carta de presentación explicando los objetivos y características de la investigación y haciendo hincapié en la importancia de su colaboración, remarcando el anonimato y que su participación en la entrevista suponía su autorización para el uso de los datos de la misma. Después de obtener los permisos correspondientes por parte de la comisión de investigación del hospital, las entrevistas se realizaron en la propia unidad, en una sala privada y después de concertar una cita previamente con el entrevistado.

Se realizo grabación de audio y posteriormente se realizó trascripción literal. La categorización y codificación de las entrevistas se realizo tras repetidas lecturas de las transcripciones y por consenso entre los investigadores.

\section{RESULTADOS}

Tras el análisis de contenido se establecieron dos categorías generales principales: expresiones sobre la realidad de las interacciones comunicativas entre los profesionales y expectativas de los profesionales y sus propuestas de mejora.

\section{- Expresiones sobre la realidad de las in-} teracciones comunicativas entre los profesionales.

Los informantes expresan mayoritariamente la opinión de que la comunicación es insuficiente tanto a nivel intraprofesional como la interprofesional, pero consideran esta última como más deficitaria. Lo que concuerda con las afirmaciones de los autores que aseguran que compartir la información y los objetivos sigue siendo una de las asignaturas pendientes y de los grandes retos de las organizaciones sanitarias.

Destacan que la comunicación informal es la más utilizada y acentúan la importancia de que existan más canales formales y más fluidos, con protocolos y documentos escritos que recojan la información relevante y necesarios también para la puesta al día y el aprendizaje de los nuevos profesionales que se incorporan a la unidad:

"La información es informal, es lo que hemos estado hablando antes, es el boca a boca, nada formal, escrito, dicho o protocolizado. Los relevos y el tratamiento médico están más o menos especificados y luego uno es como es, y escribe más o menos". (E 5)

La transmisión de esta comunicación informal se ve favorecida por las características del servicio y por la relación de confianza que se establece entre las personas que trabajan juntas habitualmente. De manera que el grupo tiene importantes funciones de "ayuda", tanto en la realización de tareas como de apoyo emocional:

"Aquí como el espacio es cerrado, estamos un montón de horas mucha gente, entonces pues claro, hasta ya casi nos sabemos todos los de todos los enfermos, aunque no lo lleves tu, pero ya sabes que aquel ha vomitado,.. ¿Yo que sé? al haber pocos enfermos, eso favorece, es más fácil. Y luego la comunicación que hay entre nosotros también, el llevarse bien, el comunicarse bien..."(E 2)

Los informantes expresan la inquietud que les provoca el no saber en ocasiones qué objetivos se han marcado los facultativos con el paciente. El conocimiento de informaciones del paciente se considera también de gran utilidad para el establecimiento de relaciones adecuadas con los familiares de estos. Reconocen que por las características del servicio y también 


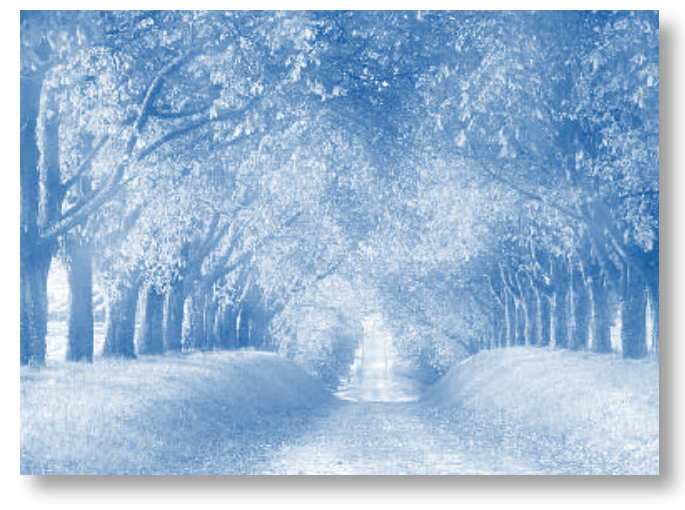

por la buena relación que en general hay con los intensivistas, mucha de esta información se solicita y es aportada, de manera informal, pero que esto depende en gran medida de las características personales tanto de enfermeros como de médicos, y de la relación personal que haya entre ellos. Se hace también referencia a la jerarquización y a las culturas profesionales, el mantener los espacios profesionales, produce en ocasiones distanciamiento y conflictos:

"Hay poca información formal. A lo mejor le preguntas al médico y se explaya, a lo mejor otro es más escueto, o te da más apuro acercarte, ya depende del carácter de cada persona y parece que te cuesta más acercarte a preguntarle. [...]. Muchas veces no sabemos el camino que van a seguir con los pacientes, no sabemos nada. Venimos hacemos los cuidados de enfermería nuestros pero no llegamos mas allá". (E 1)

Generan conflicto la existencia de rumores y de críticas y se considera que es necesario hablar sobre los errores de forma abierta y constructiva, como oportunidad para aprender y mejorar.

"Nos tenemos que comunicar. Y decirnos las cosas aunque fallemos, más que decirnos lo bueno, decirnos lo malo, para mejorarlo. [...] Lo bueno también, pero lo malo para mejorarlo, porque si no, pues lo mismo estamos cayendo siempre en lo mismo". (E6)
- Expectativas de mejora de la comunicación y propuestas de los profesionales.

En los discursos aparece recurrentemente la necesidad de crear espacios y tiempos para compartir informaciones, unificar criterios, elaborar, actualizar protocolos y realizar formación continuada. Se considera que sería positivo que las reuniones fuesen de corta duración, dentro del horario de trabajo dirigidas adecuadamente para no desviar la atención, y centradas en pocos temas claves. También se apunta que sería más eficaz hacer pequeños grupos de trabajo, para discutir temas de interés y hacer protocolos etc., y que hay que asegurar la difusión a todas las personas de los acuerdos y propuestas alcanzados de forma oral y escrita:

"Para mí lo más importante seria eso de hablar. Juntarnos más, [...] Y si hacemos una reunión, todo escrito eso se le pasa a la gente de lo que se está hablando, y que cada uno tenga una carpetica con esas cosas. $Y$ cuando venga uno nuevo también se lo das. Entonces si tuviéramos todo protocolizado o escrito, sería más fácil”. (E 5)

A nivel interprofesional expresan un gran interés por conocer más acerca de los objetivos y líneas de trabajo que se marcan los facultativos con los enfermos y que en las actividades más interdependientes se establezcan objetivos comunes, a través de sesiones y reuniones conjuntas, y también participando en la información a familiares:

"Y yo creo que hace falta unas sesiones médicas con nosotros, interdisciplinares y se lo he dicho a ellos y todo, aquí hace falta. [...] tú no sabes lo bueno que es para enfermería, trabajar así." (E1)

"Yo he oído que en otros sitios, que para informar a los familiares se va el enfermero y el médico e informan los dos. Aquí vamos ni 


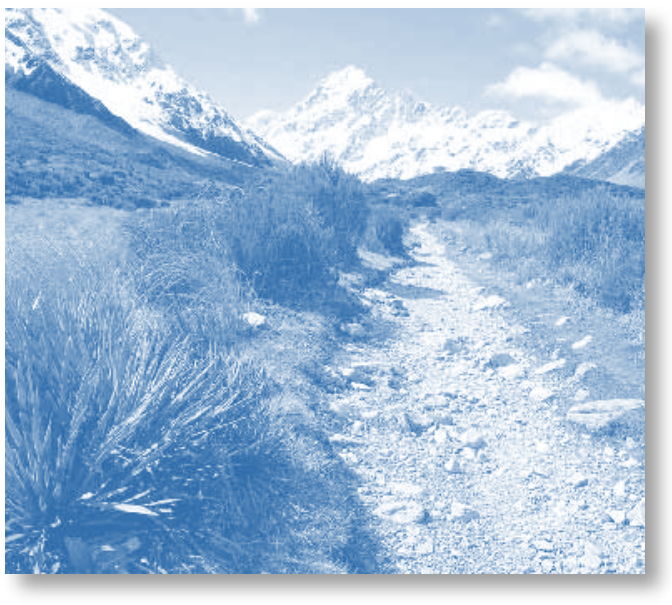

arrimados. $\mathrm{Si}$, sería positivo el que tu pudieras decirle lo que has hecho, y que él especificara. [...] Hay temas que podemos hablar con las familias y que son temas nuestros". (E 5)

Se identifica como esencial la implicación de los líderes de la unidad y de la organización, como guías, pero también como propulsores de las medidas necesarias para poder poner en funcionamiento estos cambios:

“Tendría que haber una persona más o menos guía, o varias personas, que no hace falta que sea una sola. Es verdad que hay líderes que no son jefes, que la gente se incentiva, se motiva, porque haya alguien que los motive, que motive el trabajo en equipo, y que se intente canalizar los malos rollos, crear buen ambiente. Eso ¿cómo se hace $\neg$ ? Desde mi punto de vista, hablando y hablando. (E 5)

\section{CONCLUSIONES Y DISCUSIÓN}

Hemos considerado que la investigación cualitativa era la adecuada para nuestro objeto de estudio, ya que nuestra pretensión ha sido ver como los actores daban sentido e interpretaban su realidad. Reconocemos, por tanto, que los datos no son generalizables, pero si pueden ser de gran utilidad para intervenciones concretas dentro de la unidad de estudio, ya que ponen de manifiesto la experiencia y la interpretación de los sujetos en su propio contexto (Salamanca Castro, 2006).

Las expectativas e interpretaciones de los actores está influida por diversos factores, de la propia organización, pero también autobiográficos, atribuibles a la formación, experiencias vividas y también a las características personales. Pero en las entrevistas aparecen vivencias compartidas, que hacen aflorar categorías comunes.

La comunicación es vista por los profesionales de enfermería como una herramienta imprescindible para el cuidado del paciente, pero es valorada como insuficiente en términos generales, destacando la falta de consenso, de intercambio de informaciones sobre los pacientes y las dificultades en la transmisión de nuevas técnicas y habilidades. Creen necesario mejorar la comunicación conjunta dentro del equipo para prestar unos cuidados adecuados, lo que consideran es también beneficioso para el aprendizaje interprofesional y la solución de problemas (Kvarnström, 2008; Simmons y Sherwood, 2010)

Las dinámicas comunicacionales que se establecen están influenciadas en gran medida por las relaciones jerárquicas existentes, por la percepción de los distintos roles y límites profesionales, y la ausencia de apoyo organizacional al trabajo en equipo real (Zwarenstein $M$, Reeves $S$ 2002). Cada profesión sanitaria tiene una cultura diferente, fruto de las diferencias en los ambientes de socialización y aprendizaje, que hace que cada uno tenga sus propios valores y punto de vista disciplinario de las necesidades del paciente pero también diferentes estilos de comunicación (Leonard, Graham y Bonacum, 2004). Lo que en ocasiones complica y hacen ineficaces las relaciones comunicacionales, con patrones unidireccionales y con poca reciprocidad. 
Para salvar estas barreras los autores remarcan la necesidad de establecer relaciones interdisciplinarias basadas en la confianza y el respeto mutuo (D’Amour y San Martín, 2006), $y$ en el conocimiento y reconocimiento de lo que puede aportar cada profesional, en este sentido cabe destacar la importan de la formación no solo en habilidades técnicas individuales sino también en habilidades sociales y de comunicación (Dave, Dault y Priest, 2007).

Pero el establecimiento de las relaciones de confianza depende de múltiples factores personales pero también organizacionales, entre los que juega un papel importante la alta rotación del personal de enfermería (Lucas, Gómez y Antón, 2011).

Es importante reconocer que la comunicación efectiva es compleja y está es influenciada por múltiples factores: situacionales, de personalidad, de conocimientos, de valores culturales, etc., pero fomentar la comunicación estandarizada puede ayudar a transmitir la información oportuna necesaria para asegurar unos cuidados seguros y de calidad, lo que implica el desarrollo de comunicación abierta, basada en la confianza (D’Amour y San Martín, 2006) y la relación entre los miembros del equipo para el aprendizaje, el respeto y la comprensión mutua, así como la participación en toma de decisiones y responsabilidades. Estos cambios precisan la implicación de la organización para desarrollar estrategias institucionales que favorezca la participación y que dediquen tiempo y recursos para suministrar a los individuos las herramientas necesarias para el trabajo colaborativo (Rubio et al, 2006), entre las que cabe destacara actividades de aprendizaje que promuevan la comunicación interprofesional eficaz (Dave, Dault y Priest, 2007).

Los lideres deberían aprovechar la preocupación y el interés expresados por los profesio- nales, y promover iniciativas para el intercambio y la colaboración.

\section{BIBLIOGRAFÍA}

- Arizeta, A.G. ; Portillo, I. y Ayestarán, S. (2001). Cambio Organizacional y Cultural en un Hospital: percepciones y discurso de sus impulsores. Rev Calidad Asistencial, 16:22-28.

- Baker, DP; Day, R y Salas, E. (2006). Teamwork as an essential component of high-reliability organizations. Health services research, 41 (4, part. II), 1576-1598.

- Clark P.R. (2009). Teamwork: building healthier workplaces and providing safer patient care. Critical care nursing quarterly, 32 (3): 221-231.

- Cleries X, Kronfly E (2000). El aprendizaje de habilidades de comunicación por parte de profesionales sanitarios: reflexiones a partir de diversas experiencias. Educ Med;3(1): 9-18.

- D’Amour, D., San Martín Rodríguez, L. (2006). La colaboración entre los profesionales de la salud. Contexto, dinámica y elementos determinantes. En Revista rol de Enfermería, 29(6) 425-430

- Dave C., Dault M. y Priest A (2007). Effective Teamwork in Healthcare: Research and Reality Priest HealthcarePapers, 7 (Sp): 26-34

- Gil Rodríguez, F.; Barrasa Notario, A. (2003). Comunicación en las organizaciones. En Gil Rodríguez, F. Alcover de la Hera, C.M. (Coordinadores). Introducción a la psicología de las organizaciones (pp. 321- 353). Madrid. Alianza editorial S.A.

- Glaser, B. G. y Strauss, A.L. (1980). The discovery of grounded theory : strategies for qualitative research. (11 ${ }^{\mathrm{a}}$ ed.). New York. Aldine.

- Leonard, M., Graham, S., Bonacum, D. (2004). The human factor: The critical importance of effective teamwork and communication in providing safe care. Qual Saf Health Care, 13: 85-90.

- Lucas García, C., Gómez García, C., Antón Hurtado, $J^{a}$. (2011) Culturas del trabajo y prácticas profesionales: estudio exploratorio del trabajo en equipo en la unidad de cuidados intensivos del hospital universitario 


\section{ᄃultura de las Cuidados}

Rafael Méndez de Lorca. En Andréu Abela J. y

- Pérez Corbacho AM. (eds.). Actas de la Conferencia Iberoamericana de Investigación Cualitativa. (pp.232-234). Universidad de Granada.

- Plaza Escribano, T. et al. (2009). Comunicación interna en el equipo de enfermería. Metas de enfermería, 12 (1), 8-12.

- Reader, TW., Flin, R., Mearns, K., Cuthbertson, BH. (2009). Developing a team performance framework for the intensive care unit.Critical care medicine, 37(5):1787-1793.

- Rubio Rico, L., et al. (2006). Relaciones interdisciplinarias y humanización en las unidades de cuidados intensivos. Enfermería Intensiva, 17(4):141-53.

- Salamanca Castro A. B. La investigación cualitativa en las ciencias de la salud. Nure Investigación. 2006; 24. Consultado el 1/02/2010 en: http://www.nureinvestigacion. es/ FICHEROS_ADMINISTRADOR/F_METODOLOGICA/ FMetod_24.pdf

- Salas, E. et al. (2009). What are the critical success factors for team training in health care? The Joint Commission journal on quality and patient safety, 35(8): 398-405.

- Simmons, D., Sherwood, G. (2010). Neonatal intensive care unit and emergency department nurses' descriptions of working together: building team relationships to improve safety. Crit Care Nurs Clin North Am, 22(2):253-60

- Zwarenstein, M., Reeves, S. (2002). Working together but apart: Barriers and routes to nurse-physician collaboration. Jt Comm J Qual Improv, 28: 242-247.

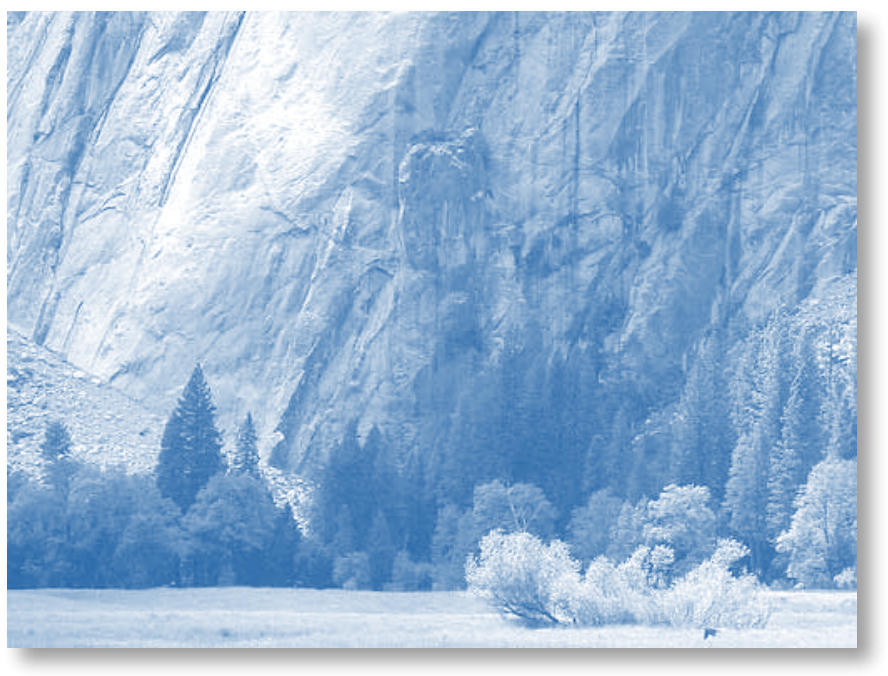

